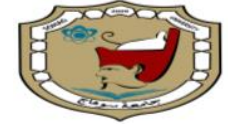

Sohag University

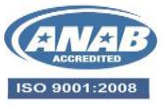

Sohag Medical Journa

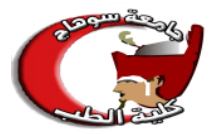

Faculty of Medicine

\title{
The tumor site of origin and its effect on survival in gastrointestinal stromal tumors (GIST)
}

\author{
Ali Mohamed Ali, Asmaa Abdelghany Abdellatif, Amal Ali Omar. \\ Clinical Oncology, Oncology department, faculty of medicine, Sohag university
}

\begin{abstract}
Background: GIST is a common mesenchymal tumor of the Gastrointestinal Tract. The stomach is the most frequently affected site. In this study, we focus on the relationship between tumor size and survival. Materials and methods: our retrospective study included 47 patients diagnosed with GIST at Sohag University Hospital, Sohag Cancer Center, and Sohag Health Insurance Hospitals during the period between 2012 -2017. The collected data were extracted from patient's files. Missed data in the files were obtained via phone communication with the patients or their relatives.

Results: 47 cases with gastrointestinal tumors were selected. All tumors were stained positive for c-kit. $48.9 \%$ (23/47) of the tumors were located in the stomach, $40.4 \%$ $(19 / 47)$ in the small intestine, and $10.6 \%(5 / 47)$ in the large intestine. The mean progression-free survival (PFS) was 20.75 months ranging between 7 months and 39 months. On analyzing the impact of tumor site on PFS we found that There was a significant relationship between the gastric site of the tumor and improved PFS (logrank $\mathrm{P}=0.010$ ). The mean overall survival (OS) was 35.26 months ranging between 1 month and 108 months, on analyzing the impact of tumor site on OS we found no relation.
\end{abstract}

Conclusion: Gastric origin of GIST is associated with more benign behavior and improved PFS in comparison with the intestinal origin.

Keywords: GIST, gastric GIST, survival.

\section{Introduction:}

GISTs are a common mesenchymal tumor of the Gastrointestinal Tract (1). Incidence varies from 10 to 15 cases per million and the median age at diagnosis is 63 years ranging between age 60 and 65 (2).

The majority of GIST (about 70\%) are found in the stomach (3), About 20\% are originated from the small intestine, About 5\% found in the large intestine and the rectum (4), And less than 5\% are found in the esophagus(5). Omental and mesenteric origin has been reported only in single cases (6).

The clinical presentation depends on the site and the size of the tumor (7). Gen- etic studies have revolutionized the management of GIST using targeted therapy (8).

A randomized phase III trial demonstrated the efficacy of adjuvant therapy with imatinib in GISTs for high-risk patients. Recurrence-free survival (RFS) was significantly prolonged when given for a duration from 1 to 3 years (9).

.In patients with unresectable, metastatic, or recurrent GISTs, Imatinib mesylate is the first-line therapy, When patients have the progressive disease under imatinib treatment or can't tolerate it imatinib due to side effects, sunitinib malate is used. Regorafenib is a tyrosine 
kinase inhibitor used for imatinib and sunitinib-resistant GIST (10).

Aim of the work: evaluating the relation between tumor site of origin and survival in patients with gastrointestinal stromal tumors at Sohag governorate in the period between 2012 and 2017.

\section{Materials and Methods:}

Patient Population: A total number of 47 patients diagnosed with GIST were enrolled in a retrospective study at the oncology department in Sohag University Hospital, Sohag Cancer Center, and Sohag Health Insurance Hospitals during the period between $2012-2017$. All patients in our retrospective study were having the following eligibility criteria.

Inclusion criteria: our study included adult male and female patients who were proved histologically to have GIST.

Exclusion criteria: patients who have co-morbidities that contraindicate treatment and patients with a history of concomitant cancers were excluded from the study.

All participants were diagnosed with GIST based on pathology, and they were categorized according to the tumor site of origin into gastric, small intestinal, or large intestinal.

All patients received their treatment in Sohag but some of them came from nearby governorates like Quan and the red sea.

\section{Methods of data extraction:}

The collected data were extracted from patient's files at the oncology department in Sohag University Hospital, Sohag Cancer Center, and Sohag Health Insurance Hospitals. Some missed data in the files were obtained via phone communication with the patients or their relatives.

Assessment of outcomes of treatment was done by means of the following endpoints:
Overall survival (OS): the period started from the date of diagnosis until patient death or the time of the last follow-up. Progression-free survival (PFS): The length of time during and after the treatment of a disease that a patient lives with disease but it does not get worse.

\section{Statistical analysis:}

Initial data exploration was done by identifying the mean, median, standard deviation, and drawing a suitable histogram to identify the data distribution. Identifying the cut-off level for scale data was done through a ROC curve. Initial hypothesis testing of quantitative data was done by the methods of comparing means (independent-samples T-Test) while for categorical data Chi-Square test was used. For those with a rejected null hypothesis, a Kaplan-Meier survival test was used to estimate the distribution of PFS and OS where the log-rank test was used to compare the difference between survival curves of the different subgroups. Significance is considered if $(\mathrm{P}<0.05)$. For significant variables, Data correlation was done by bivariate analysis then all significant variables were subjected to build a COX-Regression model.

\section{Ethical approval:}

This study was approved by the research board and the scientific ethics committee of the Sohag Faculty of Medicine.

\section{Results:}

In this retrospective study, 47 cases with gastrointestinal tumors were selected. All tumors were stained positive for c-kit. By the end of this retrospective study, 27(57.4\%) cases were alive while $14(29.8 \%)$ cases were dead and $6(12.8 \%)$ cases unknown. Among the participants in our study, $48.9 \%(23 / 47)$ of the tumors were located in the stomach, $40.4 \%(19 / 47)$ in the small intestine, and $10.6 \%(5 / 47)$ in the large 
intestine. The mean age at diagnosis was 51.11 years (SD 11.310). Most patients were diagnosed between 26 and 72 years of age. There was a slight female predominance, With $55.3 \%$ (26/47) females vs. $44.7 \%$ (21/47) males. Abdominal pain was the main symptom in $78.7 \%$ (37/47) of patients but simultaneous symptoms were common, palpable abdominal tumor in $19.1 \%$ (9), vomiting was a symptom in $55.3 \%$ (26), GIT bleeding in $6.4 \%$ (3), $17 \%$ (8) of patients were presented by intestinal obstruction and other symptoms were rare.

Fourteen patients have a disseminated disease and 6 had an irresectable tumor at presentation. Six patients received neoadjuvant imatinib two of them underwent surgery after that.

Fourteen patients $(29.8 \%)$ had metastatic disease at the time of diagnosis with the majority metastasizing to the liver (7/14), peritoneum (2/14), lungs $(2 / 14)$ or spleen (1/14), pancreas $(1 / 14),(2 / 14)$ cases were metastasizing to both liver and peritoneum.

Forty-one patients (87.2\%) were considered for surgical resection, complete negative resection margin was performed in $53.2 \%$ (25/41) of patients. In one patient the microscopic margins were recorded as positive. In 15 patients $(31.9 \%)$ pathology reports have no comment about resection margin.

Fourteen patients $(29.8 \%)$ were T2, $29(61.7 \%)$ were $\mathrm{T} 3$ and $4(8.5 \%)$ were T4. In 26 patients $(55 \%)$ no available data about side effects of imatinib, while gastritis was the most common side effect reported in 16 patients (34\%), 5 patients $(10.6 \%)$ had no side effects. The mean duration of treatment was 30.9130 months ranging between 6 months and 102 months.

The recurrent disease occurred in $4.3 \%$ (2/47) of patients, and all of these patients had had complete resection of their primary disease. Progressive disease occurred in $38.3 \%$ (18/47) of patients.

The mean PFS was 20.75 months ranging between 7 months and 39 months. On analyzing the impact of tumor site on PFS we found that There was a significant relationship between the gastric site of the tumor and PFS (log-rank P = 0.010)figure(1), table(1).

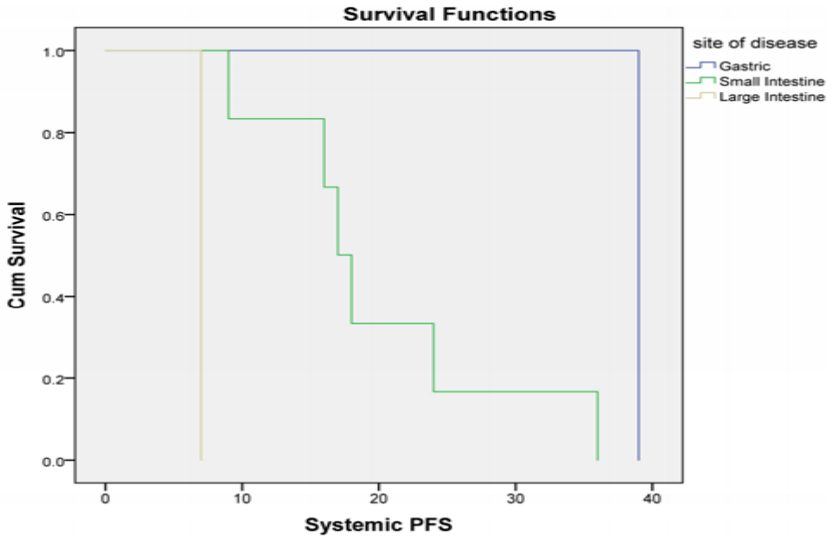

Figure (1) illustration of statistical relation between tumor site and PFS. 
SOHAG MEDICAL JOURNAL

Vol. 25 No. 2 April 2021
The tumor site of origin and its effect on survival Amal Ali Omar

\begin{tabular}{|l|l|l|l|l|l|r|l|l|}
\hline \multirow{2}{*}{$\begin{array}{l}\text { Site of } \\
\text { disease }\end{array}$} & \multicolumn{3}{|c|}{ Mean } & \multicolumn{5}{c|}{ Median } \\
\cline { 2 - 9 } & estimate & $\begin{array}{r}\text { Std. } \\
\text { error }\end{array}$ & $\begin{array}{r}\text { Lower } \\
\text { bound }\end{array}$ & $\begin{array}{r}\text { Upper } \\
\text { bound }\end{array}$ & estimate & $\begin{array}{r}\text { Std. } \\
\text { error }\end{array}$ & $\begin{array}{l}\text { Lower } \\
\text { bound }\end{array}$ & $\begin{array}{l}\text { Upper } \\
\text { bound }\end{array}$ \\
\hline $\begin{array}{l}\text { Gastric } \\
\text { Small } \\
\text { intestine }\end{array}$ & 29.000 & .000 & 39.000 & 39.000 & 39.000 & & & \\
$\begin{array}{l}\text { Large } \\
\text { intestine }\end{array}$ & 7.000 & 3.751 & 12.649 & 27.351 & 17.000 & 1.225 & 14.600 & 19.400 \\
overall & 20.750 & 4.113 & 12.688 & 28.812 & 17.000 & 1.414 & 14.228 & 19.772 \\
\hline
\end{tabular}

Table (1): mean and median of PFS in relation to the tumor site.

The mean OS according to the results of this study was 35.26 months ranging between 1 month and 108 months on analyzing the impact of tumor site on OS no effect was found in figure(2), table(2).

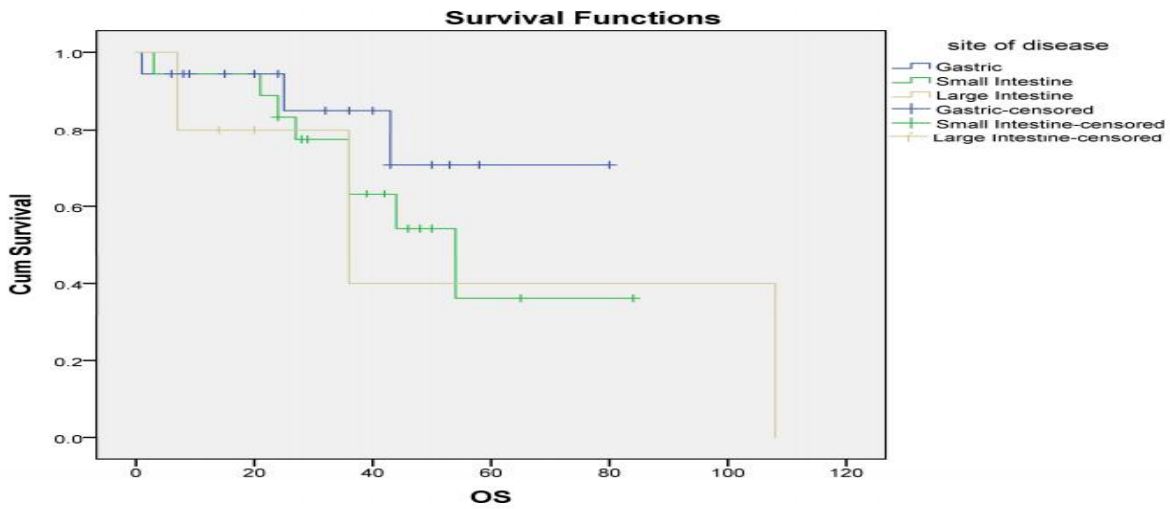

Figure(2) illustration of statistical relation between tumor site and OS.

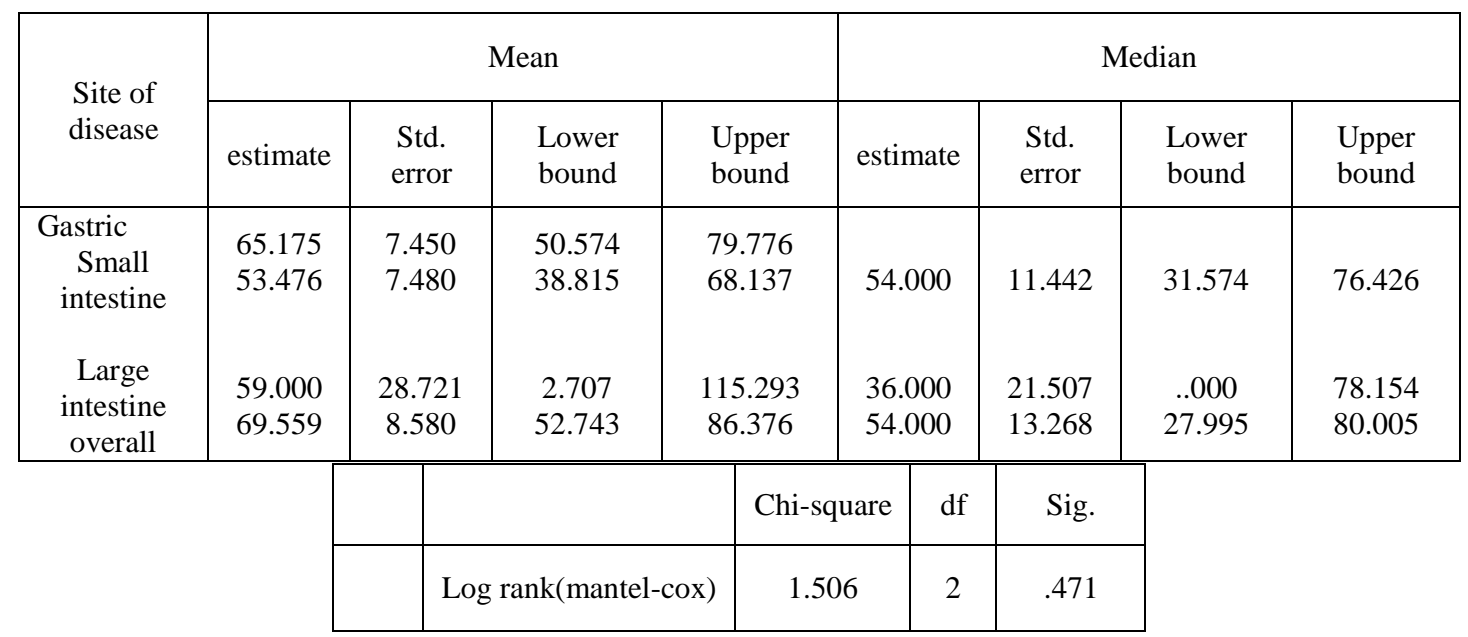

Table (2)mean and median of OS in relation to the tumor site. 


\section{Discussion:}

In our study, a total number of 47 patients diagnosed with GIST were enrolled in a retrospective study on treatment outcomes of GIST at the oncology department in Sohag University Hospital, Sohag Cancer Center, and Sohag Health Insurance Hospitals.

According to our study, the most common site of the tumor was the stomach (48.9\%), 40.4\% (19/47) in the small intestine, and $10.6 \%(5 / 47)$ in the large intestine. This result is similar to results reported from Dougherty et al (11) in which the gastric site accounted for $83 \%$ of cases. According to Aghdassi et al, the most common GIST location was the stomach (67.6\%) (12). A recent study reported showed a similar pattern of distribution of GIST with $61 \%$ arose in the stomach (13). In a study conducted by Barakat et al, about $41 \%$ of GISTs in a Jordanian study population located in the stomach (14). More than half of GIST cases in the Egyptian study reported by Sorour et al originated from the stomach (15). According to Alghamdi et al, The most frequently affected organs were the stomach (63.8\%) (16). According to Bokhary et al, $51.4 \%$ of GIST cases in the Saudi Arabian study originated from the stomach (17). According to Kassem et al, (54\%) of patients have tumors originated from the stomach (18).

In our findings even though $48.9 \%$ of the tumors were located in the stomach, 9 of 14 metastatic tumors were non-gastric. This could indicate that gastric GIST is an inherently more benign disease. This finding is in line with many other studies which suggested an inherent biological difference between gastric and non-gastric tumors as reported by Lasota et al (19), and Tryggvason et al (5).

Patients in our study most frequently presented with abdominal pain (78.7\%). This finding is consistent with Al-Thani et al, in which abdominal pain was pres- ent in most of the patients (85\%) (20), and Alghamdi et al,(16). In most reports, gastrointestinal bleeding was the most frequent clinical manifestation as reported by Miettinen et al, (21), Aghdassi et al, (12), and Zhao et al, (7). In our study 14 patients $(29.8 \%)$ have metastatic disease at the time of diagnosis with half of them had metastasis in the liver (7/14), this is matched with Aghdassi et al, where the liver is the most frequent site of metastases (12). .In our study 14 patients $(29.8 \%)$ have metastatic disease at the time of diagnosis with the majority metastasizing to the liver (7/14), Nine of the 14 patients who developed metastases have their initial tumor originating from nongastric site namely the small intestine, This could indicate that the gastric site of GIST is associated with a better outcome. According to Aghdassi et al, the liver being the most frequent site of metastases (12).

Many authors in the literature confirm that surgical resection is still the gold standard treatment for GIST, especially when localized, allowing to reach a cumulative 5 years survival of almost $50 \%$ (22). In our study 41 (87.2\%) of patients were considered for surgical resection, a complete negative resection margin was performed in $53.2 \%(25 / 41)$ of patients. In one patient the microscopic margins were recorded as positive. In 15 patients (31.9\%) pathology reports have no comment about resection margin. Fourteen patients have a disseminated disease and 6 have an irresectable tumor at presentation, six patients received neoadjuvant imatinib two of them underwent surgery after that.

All cases in this study were treated with imatinib, six received neoadjuvant treatment, and 41 received adjuvant treatment among them 41 patients were regular on treatment and 6 patients were irregular. 
The incidence of lymph node involvement in our series was $0 \%$, which is similar to the results of Di Vita et al,(23), And consistent with a low incidence of lymph node metastases seen in other reports (less than 2\%) as reported by Burkill et al, (24) and Sawaki \& Yamao, et al, (25).

In our study, the mean PFS was 20.75 months ranging between 7 months and 39 months. The PFS was better in patients with gastric site primary which is similar to the results of Di Vita et al, (23), and Alghamdi et al,(16). Lillemoe et al, in a study of 133 patients with resected GIST also reported that survival was related to the tumor's site (26). According to Kassem et al. patients with gastric lesions had a better prognosis than did patients with lesions in other sites (18). stated that anatomic location is a prognostic factor independent of tumor size, mitotic rate, and patient's age, with a trend for small bowel tumors to have the worst prognosis (27), Emory and Sobin et al, also reported that anatomic site was related to the prognosis of GIST with gastric GIST having the best outcome (28). Lin, et al. found that most of their patients with small intestinal GIST had lesions larger than $5 \mathrm{~cm}$ and a poorer outcome than those with gastric tumors (29).

In our study the mean OS was 35.26 months ranging between 1 month and 108 months. in our study the OS is not proved to be affected by tumor site, many studies showed improved OS in gastric site GIST as reported by Di Vita et al (23).

The limitations of the study: Multiple limitations were present in our study and these limitations may affect the results of the study; as GIST is a rare disease, so a small number of patients included in our retrospective study, lack of surgical details in patient's files, defect in proper genetic studies due to fina- ncial issues, unavailability of second and third lines targeted therapy and that makes our study only comparable with limited studies, defect in proper reporting of data in patient's files.

\section{A list of abbreviations: \\ GISTgastrointestinal stromal tumors \\ OS overall survival \\ PFS progression-free survival \\ SD standard deviation}

In our retrospective study, the outcome of treatment was strictly related to the site, Gastric origin of GIST was associated with more benign behavior and improved PFS in comparison with the intestinal origin that was in agreement with most previous studies.

\section{References:}

1. Goettsch, W. G., Bos, S. D., Breekveldt-Postma, N., Casparie, M., Herings, R. M., \& Hogendoorn, P. C.et al . Incidence of gastrointestinal stromal tumors is underestimated: results of a nation-wide study. Eur $J$ Cancer,2005; 41(18): 2868-2872.

2. Ortenzi M, Ghiselli R, Cardinali L, Guerrieri M: Surgical treatment of gastric stromal tumors: Laparoscopic versus open approach. Ann Ital Chir. 2017;88. PII: S0003469X17026112.

3. Nilsson, B., Bumming, P., MeisKindblom, J. M., Oden, A., Dortok, A., Gustavsson, B., Sablinska, K., \& Kindblom, L. G. et al. Gastrointestinal stromal tumors: the incidence, prevalence, clinical course, and prognostication in the imatinib mesylate era--a population-based study in western Sweden. Cancer 2005; 103(4): 821-829.

4. Liu, Z., Wu, S., Gou, S., Li, Y., Wu, H., \& Li, Y.et al . A Population-Based Study of the Incidence and Survival of Anorectal Gastrointestinal Stromal Tumor. Medical science monitor: international medical journal of experimental and clinical research 2019; 25: 5408-5417. 
5. Tryggvason, G., Gislason, H. G., Magnusson, M. K., \& Jonasson, J. G. et al. Gastrointestinal stromal tumors in Iceland, 1990-2003: the Icelandic GIST study, a population-based incidence, and pathologic risk stratification study. Int J Cancer,2005; 117(2): 289-293.

6. Lee $\mathrm{YH}$, Chong GO, Hong DG. Is gastrointestinal stromal tumor (GIST) originating from the rectovaginal septum GIST or extraGIST (EGIST)? A case report with literature review. Eur J Gynaecol Oncol. 2015; 36: 7504.

7. Zhao, Y., Li, K. K., Ng, K. P., Ng, C. H., \& Lee, K. A.et al. The RNA Pol II sub-complex hsRpb4/7 is required for the viability of multiple human cell lines. Protein Cell 2012; 3(11): 846854.

8. Miettinen, M., \& Lasota, J. Histopathology of a gastrointestinal stromal tumor. J Surg Oncol,2011; 104(8): 865-873.

9. Ahmed, M.. Recent advances in the management of gastrointestinal stromal tumor. World journal of clinical cases,2020 8(15): 3142-3155. .

10.Rutkowski P, Przybył J, Wozniak A, Badalamenti G. Targeted therapy in gastrointestinal stromal tumours. Target Ther Solid Tumors Curr Clin Pathol,2015;163-196.

11. Dougherty, M. J., Compton, C., Talbert, M., \& Wood, W. C. Sarcomas of the gastrointestinal tract. Separation into favorable and unfavorable prognostic groups by mitotic count. Annals of surgery,1991a; 214(5): 569.

12.Aghdassi, A., Christoph, A., Dombrowski, F., Döring, P., Barth, C., Christoph, J., Lerch, M. M., \& Simon, P.et al. Gastrointestinal Stromal Tumors: Clinical Symptoms, Location, Metastasis Formation, and Associated Malignancies in a Single Center Retrospective Study. Digestive Diseases, 2018; 36(5): 337-345.

13. Yang, Z., Zheng, R., Zhang, S., Zeng, H., Li, H., \& Chen, W.et al. Incidence, distribution of histological subtypes and primary sites of soft tissue sarcoma in China. Cancer biology \& medicine, 2019; 16(3): 565-574.

14.Barakat, F. H., Haddad, H. A., Matalka, II, Al-Orjani, M. S., Al-Masri, M. M., \& Sughayer, M. A.et al. Characteristics of gastrointestinal stromal tumors in a Middle Eastern population. Saudi Med J,2010: 31(7): 797-802.

15.Sorour, M. A., Kassem, M. I., Ghazal, A. E.-H. A., El-Riwini, M. T., \& Nasr, A. A.et al. Gastrointestinal stromal tumors (GIST) related emergencies. International Journal of Surgery, 2014; 12(4): 269-280.

16.Alghamdi, H. M., Amr, S. S., Shawarby, M. A., Sheikh, S. S., Alsayyah, A. A., Alamri, A. M., Ismail, M. H., Almarhabi, A., Alrefaee, M. A., \& Ahmed, M. I.et al. Gastrointestinal stromal tumors. A clinicopathological study. Saudi medical journal,2019; 40(2): 12.1ץ--4

17.Bokhary, R. Y., \& Al-Maghrabi, J. A. Gastrointestinal stromal tumors in western Saudi Arabia. Saudi Med J,2010; 31(4): 437-441.

18.Kassem, M., Elzeiny, M., \& Elhaddad, H. Management of gastrointestinal stromal tumors: a prospective and retrospective study. The Egyptian Journal of Surgery, 2016; 35(1): 11-19

19.Lasota, J., \& Miettinen, M. Clinical significance of oncogenic KIT and PDGFRA mutations in gastrointestinal stromal tumours. Histopathology,2008; 53(3): 245-266.

20.Al-Thani, H., El-Menyar, A., Rasul, K. I., Al-Sulaiti, M., El-Mabrok, J., Hajaji, K., Elgohary, H., \& Tabeb, A. et al. Clinical presentation, management and outcomes of gastrointestinal stromal tumors. International Journal of Surgery,2014; 12(10): 1127-1133

21.Miettinen, M., Kopczynski, J., Makhlouf, H. R., Sarlomo-Rikala, M., Gyorffy, H., Burke, A., Sobin, L. H., \& Lasota, J. et al. Gastrointestinal stromal tumors, intramural leiomyomas, and leiomyosarcomas in the duodenum: a clinicopathologic, immunohistochemical, 
and molecular genetic study of 167 cases. Am J Surg Pathol,2003; 27(5): 625-641.

22.El-Zohairy, M., Khalil el, S. A., Fakhr, I., El-Shahawy, M., \& Gouda, I. et al. Gastrointestinal stromal tumor (GIST)'s surgical treatment, NCI experience.

J Egypt Natl Canc Inst,2005; 17(2): 56-66.

23.Di Vita, M., Zanghì, A., Cavallaro, A., Cardì, F., Uhlig, M., Ursi, P., Lomenzo, E., Panebianco, V., \& Cappellani, A. et al. Gastric GIST and prognostic models. Which is the best to predict survival after surgery? Annali italiani di chirurgia ,2019; 90: 31-40.

24.Burkill, G. J., Badran, M., Al-Muderis, O., Meirion Thomas, J., Judson, I. R., Fisher, C., \& Moskovic, E. C.et al. Malignant gastrointestinal stromal tumor: distribution, imaging features, and pattern of metastatic spread . Radiology,2003; 226(2): 527-532.

25.Sawaki, A., \& Yamao, K. Imatinib mesylate acts in metastatic or unresectable gastrointestinal stromal tumor by targeting
KIT receptors--a review. Cancer Chemother Pharmacol, 2004;54 Suppl 1: S44-49.

26.Lillemoe KD, Efron DT. Gastrointestinal stromal tumors. In Current surgical therapy. Cameron JL, Gery L, editor. Mosby Inc, USA. 2001, pp. 112-117.

27.Joensuu, H., Fletcher, C., Dimitrijevic, S., Silberman, S., Roberts, P., \& Demetri, G.et al. Management of malignant gastrointestinal stromal tumours. Lancet Oncol,2002; 3(11): 655-664.

28.Emory, T. S., Sobin, L. H., Lukes, L., Lee, D. H., \& O'Leary, T. J. et al. Prognosis of gastrointestinal smooth-muscle (stromal) tumors: dependence on anatomic site. Am J Surg Pathol,1999; 23(1): 82-87.

29.Lin, S.-C., Huang, M.-J., Zeng, C.-Y., Wang, T.-I., Liu, Z.-L., \& Shiay, R.-K.et al. Clinical manifestations and prognostic factors in patients with gastrointestinal stromal tumors. World journal of gastroenterology, 2003; 9(12): 2809-2812. 